\title{
Urinary Angiotensinogen Is Elevated in Patients with Nephrolithiasis
}

\author{
Wei Sun, ${ }^{1}$ Yuan Feng, ${ }^{2}$ Xu-Dong Yao, ${ }^{1}$ Yun-Fei Xu, ${ }^{1}$ Bo Peng, \\ Min Liu, ${ }^{1}$ and Jun-Hua Zheng ${ }^{1}$ \\ ${ }^{1}$ Department of Urological Surgery, Shanghai Tenth People's Hospital, Tongji University, Shanghai 200072, China \\ ${ }^{2}$ Department of Nephrology, Affiliated Drum Tower Hospital, Nanjing University, Nanjing, Jiangsu 210093, China
}

Correspondence should be addressed to

Min Liu; kidneyliu@163.com and Jun-Hua Zheng; zhengjh0471@sina.com

Received 4 February 2014; Accepted 19 March 2014; Published 9 April 2014

Academic Editor: John J. Gildea

Copyright (C) 2014 Wei Sun et al. This is an open access article distributed under the Creative Commons Attribution License, which permits unrestricted use, distribution, and reproduction in any medium, provided the original work is properly cited.

Background. Elevated urinary angiotensinogen (UA) was identified as novel prognostic biomarker capable of predicting chronic kidney disease, and in the present study, we will investigate the diagnostic value of UA in the patients of nephrolithiasis. Methods. Urine angiotensinogen levels and $\alpha 1$-microglobulin were measured by enzyme-linked immunosorbent assay (ELISA) in 60 patients presenting with nephrolithiasis and 50 sex- and age-matched healthy volunteers. Estimated glomerular filtration (eGFR) was calculated and, by simple regression analysis, the correlation of UA/ $\alpha 1$-microglobulin levels and the decline of eGFR were analyzed as well. Results. Median UA levels was significantly increased in the nephrolithiasis patients compared with normal control (1250.78 \pm 439.27 versus $219.34 \pm 45.27 \mathrm{pg} / \mathrm{mL} ; P<0.01)$. The mean serum creatinine levels in patients with higher UA levels $(>1250 \mathrm{pg} / \mathrm{mL})$ was significantly higher than those with lower UA levels $(<1250 \mathrm{pg} / \mathrm{mL})[92.23 \pm 18.13 \mu \mathrm{mol} / \mathrm{L}$ versus $70.07 \pm 11.17 \mu \mathrm{mol} / \mathrm{L} ; P<0.05]$. According to the single variate analysis, UA levels were significantly and positively correlated with urinary $\alpha 1$-microglobulin $\left(r=0.733 ; P=1.33 \times 10^{-15}\right)$, while they were significantly and negatively correlated with eGFR $\left(r=-0.343 ; P=1.03 \times 10^{-4}\right)$. Conclusion. Urinary UA is a novel biomarker for patients with nephrolithiasis, which indicates renal tubular injury. Further study on the molecular pathogenic mechanism of UA and larger scale of clinical trial is required.

\section{Introduction}

Nephrolithiasis is a condition involving the development of stones in the kidney; it is a common disease with a prevalence of $5-8 \%$ worldwide [1]. The exact cause and etiology of nephrolithiasis remain unclear. The risk factors for developing nephrolithiasis include genetics, age, sex, geography, seasonal factors, diet, and occupations [2]. No specific predictive biomarker for the disease comes on the scene and many patients are diagnosed late after marked symptoms such as renal colic and hematuria appear. A reliable biomarker for nephrolithiasis which could predict earlier diagnosis, treatment, or better monitoring is greatly demanded.
Urine is supersaturated with oxalate ions and calcium which promotes crystals formation, and then crystals grow into larger ones; aggregation and retention of crystals are important factors of nephrolithiasis formation. During the procedure, the role of renin-angiotensin system (RAS) in kidney tissue was identified recently [3]. Moonen et al. reported that the levels of blood angiotensin-converting enzyme (ACE) were significantly increased in idiopathic hypercalciuric renal stone formers compared with healthy volunteers [4].

As already known, renin is made in the juxtaglomerular apparatus and released into the interstitial space, from where it may reach the circulation via diffusion across the peritubular capillaries. Proximal tubular fluid, however, also 
contains renin, suggesting that circulating renin is filtered in the kidney [5]. Circulating, liver-derived angiotensinogen (Ang) diffuses into the interstitium, reaching interstitial fluid levels that are comparable to those in blood [6]. Circulating ACE plays little, if any, role, and, thus, renal Ang II generation will depend entirely on locally expressed, membrane-bound ACE in the kidney [7]. Indeed, in human kidney, ACE is abundant in the brush border of the proximal tubule and, remarkably, usually absent in endothelial cells of any vessel type [8]. Endothelial neoexpression of ACE comes into play in different diseases, for example, diabetes mellitus and chronic arterial hypertension [9].

Recent interest focuses on the occurrence of both renin and angiotensinogen in urine, as markers of renal reninangiotensin system (RAS) activity, potentially reflecting the disease state [10]. Elevated urinary angiotensinogen (UA) was identified as novel prognostic biomarker capable of predicting adverse outcomes in worsening of acute kidney injury and even need of hemodialysis therapy in intensive care unit [11]. However, there were no studies that examined in detail the urinary angiotensinogen in the patients of nephrolithiasis. In the present study, we compared urinary angiotensinogen levels between the patients with nephrolithiasis and healthy controls.

\section{Materials and Methods}

2.1. Patients. We conducted a case control study between January 2012 and March 2013 at Tongji University tenth peoples' hospital. Patients who were diagnosed as having nephrolithiasis during the period were enrolled in this study. Briefly, they were diagnosed by ultrasonography and radiography. No case was found by X-ray to have radiolucent stones or by clinical evaluation to have cystine or uric acid stones. If stone specimens were removed by surgery or obtained after medical treatment or shock-wave lithotripsy, composition of the stones was confirmed by infrared spectroscopy (Spectrum RX I Fourier Transform-Infrared System, Perkin-Elmer, USA) [12].

Normal controls were randomly selected from subjects receiving general health examinations at the same hospital during the same period. The controls had no past history of nephrolithiasis and no clinical findings of stones, which were confirmed by plain abdominal X-ray and abdominal ultrasound. Both cases and controls were excluded if they had a history of chronic urinary tract infection, renal failure, chronic diarrhea, gout, renal tubular acidosis, autoimmune diseases, primary and secondary hyperparathyroidism, and cancer. All study subjects were living in Shanghai. The study protocol was approved by the Institutional Review Board of Tongji University School of Medicine. Each subject provided signed informed written consent.

2.2. Samples Collection and Assays. Since the literature reported that urinary angiotensinogen significantly correlated with decline of estimated glomerular filtration (eGFR), thus we measured fasting serum levels of creatinine $(\mathrm{Cr})$ in each patient (Wako Pure Chemical Industries, Osaka, Japan); and the eGFR was calculated by the following equations [13]:

$$
\begin{aligned}
& \operatorname{eGFR}\left(\mathrm{mL} / \mathrm{min} / 1.73 \mathrm{~m}^{2}\right)=194 \times \mathrm{Cr}^{-1.094} \times \operatorname{age}^{-0.287} \\
& \text { in males, } \\
& \operatorname{eGFR}\left(\mathrm{mL} / \mathrm{min} / 1.73 \mathrm{~m}^{2}\right)=194 \times \mathrm{Cr}^{-1.094} \times \operatorname{age}^{-0.287} \\
& \times 0.739 \text { in females. }
\end{aligned}
$$

Urinary excretions of $\alpha 1$-microglobulin and angiotensinogen were measured with enzyme-linked immunosorbent assay (ELISA) kits, LZ Test Eiken $\alpha 1$-M (Eiken Chemical Co., Tokyo, Japan) and Human Total Angiotensinogen Assay Kit (Immuno-Biological Laboratories Co., Ltd., Fujioka, Gunma, Japan).

2.3. Statistical Analysis. Data obtained by measurements were given as mean \pm standard deviation. Urinary levels of angiotensinogen, $\alpha 1$-microglobulin, and other parameters were compared by the Mann Whitney $U$ test. The concentrations of urinary angiotensinogen were compared by Student's paired $t$-test. SPSS software (version 17.0, SPSS Inc., Chicago, IL, USA) was used for statistical analyses, and $P<0.05$ was considered statistically significant.

\section{Results}

This study included 60 nephrolithiasis patients and 50 normal healthy people in the control group. The mean $( \pm S D)$ of age of patients in the study and control groups was $45.5 \pm 14.3$ and $43.6 \pm 12.7$ years, respectively. The ratio of male/female was $37 / 23$ in the study group and $35 / 15$ in the control group. The demographic characteristics of both groups are summarized in Table 1. There were no significant differences in these parameters between patients and control. Calcium oxalate and calcium phosphate were the most prevalent in the nephrolithiasis patients $(83 \%)$. Urine characteristics of both groups, including urine volume, $\mathrm{pH}$, and uric acid, were listed in Table 1, as well. The mean urinary angiotensinogen levels were shown in the last line in Table 1. The UA concentration in nephrolithiasis patients was $1250.78 \pm 439.27 \mathrm{pg} / \mathrm{mL}$, which was significantly higher than in normal control group $(219.34 \pm 45.27 \mathrm{pg} / \mathrm{mL} ; P<$ $0.01)$; the median $24 \mathrm{~h}$ urinary angiotensinogen excretion was significantly higher in nephrolithiasis patients versus normal control $(8.7 \pm 1.12$ versus $4.1 \pm 0.46 \mu \mathrm{g} / 24 \mathrm{~h} ; P<$ $0.01)$; urinary angiotensinogen-to-creatinine ratio was also significantly higher in nephrolithiasis patients versus normal control $(18.9 \pm 5.22$ versus $4.3 \pm 1.60 \mu \mathrm{g} / \mathrm{g} ; P<0.01)$, as demonstrated in Table 1 and Figure 1.

To compare the potential effect of UA on the renal function, we divided the patients into two groups according to their UA levels: UA $<1000 \mathrm{pg} / \mathrm{mL}$ and UA $>1000 \mathrm{pg} / \mathrm{mL}$, and we compared their mean serum creatinine and found that the serum creatinine levels with $\mathrm{UA}<1250 \mathrm{pg} / \mathrm{mL}$ were $70.07 \pm 11.17 \mu \mathrm{mol} / \mathrm{L}$; however, serum creatinine levels were 
TABLE 1: General characteristics of nephrolithiasis patients and healthy controls.

\begin{tabular}{lcc}
\hline Factor & Nephrolithiasis $(n=60)$ & Healthy controls $(n=50)$ \\
\hline Age (years) & $45.5 \pm 14.3$ & $43.6 \pm 12.7$ \\
Gender (male/female) & $37 / 23$ & $35 / 15$ \\
Body weight $(\mathrm{kg})$ & $62.68 \pm 11.86$ & $65.64 \pm 9.52$ \\
Height $(\mathrm{m})$ & $1.68 \pm 0.10$ & $1.70 \pm 0.08$ \\
Body mass index $\left(\mathrm{kg} / \mathrm{m}^{2}\right)$ & $22.58 \pm 5.97$ & $23.05 \pm 4.29$ \\
Urine volume $(\mathrm{mL} / 24 \mathrm{~h})$ & $1487 \pm 531.8$ & $1158 \pm 498.7$ \\
Specific gravity & $1021 \pm 7.8$ & $1023 \pm 8.4$ \\
Urine creatinine $(\mathrm{g} / 24 \mathrm{~h})$ & $1.7 \pm 0.8$ & $1.6 \pm 0.9$ \\
Urine pH & $6.21 \pm 0.56$ & $6.09 \pm 0.72$ \\
Urine uric acid $(\mathrm{mg} / 24 \mathrm{~h})$ & $667 \pm 153$ & $679 \pm 144$ \\
Urine angiotensinogen $(\mathrm{pg} / \mathrm{mL})$ & $1250.78 \pm 439.27^{*}$ & $219.34 \pm 45.27$ \\
$24 \mathrm{~h}$ UA excretion $(\mu \mathrm{g} / 24 \mathrm{~h})$ & $8.7 \pm 1.12^{*}$ & $4.1 \pm 0.46$ \\
UA/urine creatinine ratio $(\mu \mathrm{g} / \mathrm{g})$ & $18.9 \pm 5.22^{*}$ & $4.3 \pm 1.60$ \\
\hline
\end{tabular}

${ }^{*} P<0.01$ in patients compared with healthy controls.

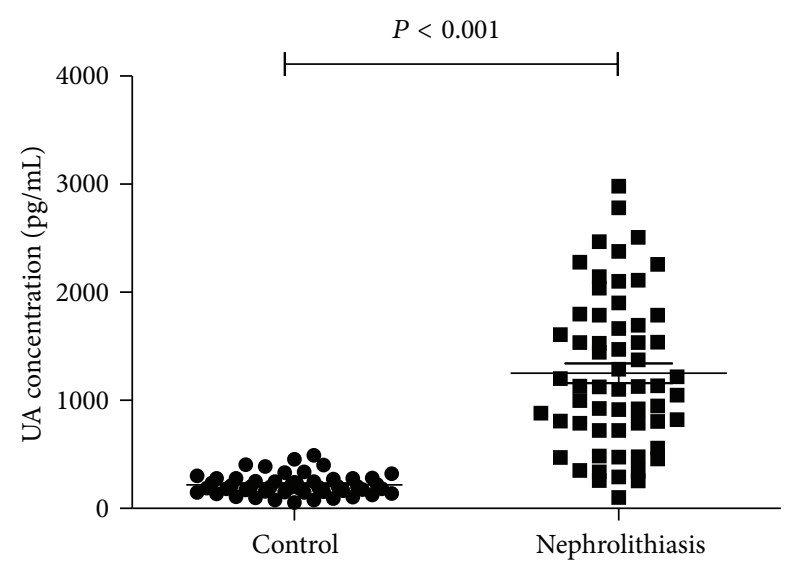

Figure 1: Urinary angiotensinogen (UA) concentration of nephrolithiasis patients and normal control. The UA concentration of nephrolithiasis patients was $1250.78 \pm 439.27 \mathrm{pg} / \mathrm{mL}$ and was of significant statistical significance compared with the control group $(219.34 \pm 45.27 \mathrm{pg} / \mathrm{mL} ; P<0.001)$.

significantly higher $(92.23 \pm 18.13 \mu \mathrm{mol} / \mathrm{L})$ in the nephrolithiasis patients with UA $>1250 \mathrm{pg} / \mathrm{mL}(P<0.05)$, which was demonstrated in Figure 2.

By simple regression analysis of the parameters, urinary angiotensinogen levels were significantly and positively correlated with urinary $\alpha 1$-microglobulin $(r=0.733 ; P=1.33 \times$ $\left.10^{-15}\right)$, while they were significantly and negatively correlated with eGFR $\left(r=-0.343 ; P=1.03 \times 10^{-4}\right)$ (Table 2 and Figure 3).

\section{Discussion}

Our study investigated the urinary angiotensinogen levels in nephrolithiasis patients and initially found that the UA concentrations in the patients with nephrolithiasis were significantly higher than those in normal subjects. In spite of normal renal function parameters in nephrolithiasis patients,

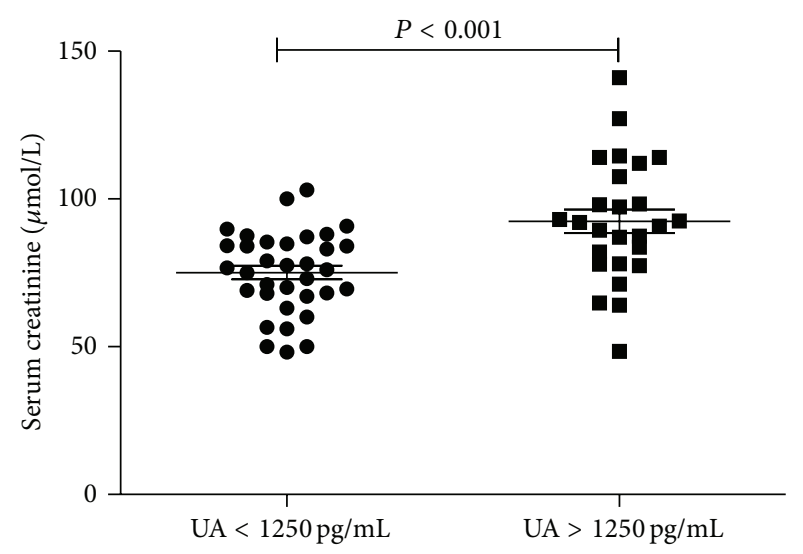

FIGURE 2: Serum creatinine levels in nephrolithiasis patients. The serum creatinine concentration of nephrolithiasis patients with UA $<1250 \mathrm{pg} / \mathrm{mL}$ was $70.07 \pm 11.17 \mu \mathrm{mol} / \mathrm{L}$ and was of significant statistical significance compared with nephrolithiasis patients with $\mathrm{UA}>1250 \mathrm{pg} / \mathrm{mL}(92.23 \pm 18.13 \mu \mathrm{mol} / \mathrm{L} ; P<0.05)$.

the mean serum creatinine levels were significantly higher in patients versus normal control. In addition, we performed a simple regression analysis and demonstrated that UA levels significantly and positively correlated with urinary $\alpha 1$-microglobulin and UA levels significantly and negatively correlated with eGFR, which suggests potential renal tubular injury in nephrolithiasis patients.

In the past studies, it has been suggested that urinary excretion of angiotensinogen reflects intrarenal angiotensin II levels $[14,15]$. Angiotensin II is a central mediator of renal injury because of its ability to produce glomerular capillary hypertension that results in damage to glomerular epithelial, endothelial, and mesangial cells [16]. Furthermore, angiotensin II and aldosterone have several nonhemodynamic effects that are also important in the pathogenesis of chronic kidney disease, including activation of pathways associated with inflammation, fibrosis, extracellular matrix 


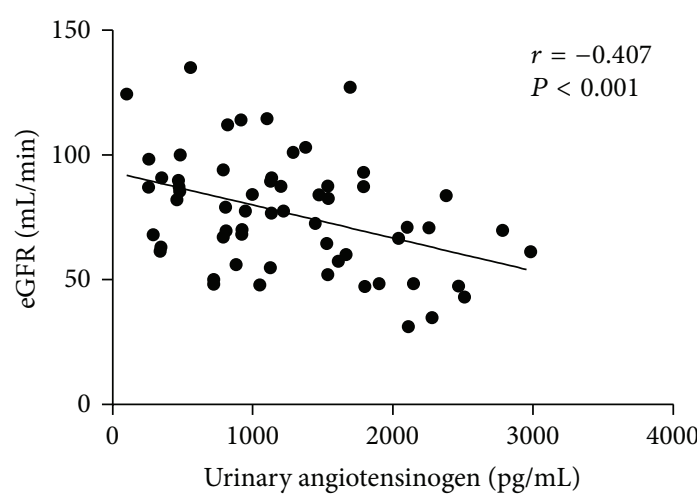

(a)

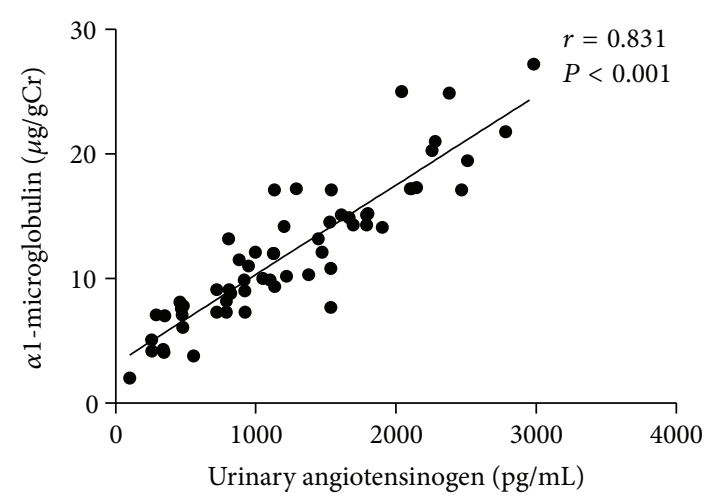

(b)

FIGURE 3: Simple correlation of urinary angiotensinogen concentration with eGFR and urinary $\alpha 1$-microglobulin in patients with nephrolithiasis $(n=60)$. (a) Estimated glomerular filtration ratio, eGFR $(r=-0.407, P<0.001)$; (b) urinary $\alpha 1$-microglobulin $(r=0.831$; $P<0.001)$. Note: Spearman correlation coefficients were used.

TABLE 2: Simple correlation of urinary angiotensinogen with various clinical parameters in patients with nephrolithiasis $(n=60)$.

\begin{tabular}{lc}
\hline & Angiotensinogen $(\mathrm{pg} / \mathrm{mL})$ \\
\hline Age $($ years $)$ & $r=-0.041 ; P=0.754$ \\
BMI $\left(\mathrm{kg} / \mathrm{m}^{2}\right)$ & $r=-0.080 ; P=0.517$ \\
SBP $(\mathrm{mmHg})$ & $r=-0.031 ; P=0.815$ \\
DBP $(\mathrm{mmHg})$ & $r=-0.182 ; P=0.127$ \\
Cr $(\mu \mathrm{mol} / \mathrm{L})$ & $r=0.261 ; P=0.021^{*}$ \\
UN $(\mu \mathrm{mol} / \mathrm{L})$ & $r=0.397 ; P=0.002^{* *}$ \\
Urine uric acid $(\mathrm{mg} / 24 \mathrm{~h})$ & $r=0.077 ; P=0.538$ \\
$\alpha 1-$ microglobulin $(\mu \mathrm{g} / \mathrm{gCr})$ & $r=0.831 ; P<0.001^{* *}$ \\
eGFR $(\mathrm{mL} / \mathrm{min})$ & $r=-0.407 ; P<0.001^{* *}$ \\
\hline
\end{tabular}

${ }^{*} P<0.05 ;{ }^{* *} P<0.01$. Spearman correlation coefficients are used.

BMI: body mass index; Cr: serum creatinine; DPB: diastolic blood pressure; eGFR: estimated glomerular filtration ratio; SBP: systolic blood pressure; $\mathrm{UN}$ : serum urea nitrogen.

accumulation, reactive oxygen species, and endothelial dysfunction [17]. As already known, the etiology of renal calculi is closely related to acute/chronic renal tubular injuries and subsequent crystal deposition and Randall's plaque formation. However, whether UA is elevated or decreased in kidney stone patients, no relative studies emerged until our present investigation.

In Alge et al.s study, urinary angiotensinogen could be useful as a prognostic acute kidney injury biomarker in the setting of the intensive care unit; in a recent study that used a definition of prerenal azotemia, which was very similar to Alge et al.s, cystatin $\mathrm{C}$, neutrophil gelatinase-associated lipocalin (NGAL), IL-18, and kidney injury molecule 1 (KIM)-1 were elevated in ICU patients with prerenal AKI compared to those without AKI but were lower than values for patients whose AKI did not resolve within $48 \mathrm{hrs}$ [18]. An important limitation of our study is that it was a relatively small retrospective biomarker qualification study with 60 nephrolithiasis patients. Larger studies will be needed to confirm the prognostic ability of angiotensinogen in this population.

In humans, plasma angiotensinogen reaches urine via glomerular filtration, like albumin, and the normal urinary angiotensinogen levels in humans are $\sim 0.2 \mathrm{pmol} / \mathrm{mL}$ versus $\sim 1,200 \mathrm{pmol} / \mathrm{mL}$ in plasma. Thus, the urinary angiotensinogen levels range from $0.01 \%$ to $0.1 \%$ of the plasma levels in humans. In contrast, urinary angiotensinogen levels in rodents are much higher and range from 0.1 to $400 \mathrm{pmol} / \mathrm{mL}$, implying that the urinary angiotensinogen levels in rodents are sometimes higher than their plasma levels [19]. Less than 100-fold higher urinary angiotensinogen levels in rodents suggested the concept that urinary angiotensinogen is exclusively plasma-derived in humans, whereas it reflects angiotensinogen release from renal tissues, possibly proximal tubules, in rodents [20].

Urinary $\alpha 1$-microglobulin is filtered freely through glomerular capillaries and reabsorbed by the proximal tubules [21]. Thus, urinary $\alpha 1$-microglobulin is a marker for proximal tubule dysfunction, and increased levels of urinary $\alpha 1$-microglobulin have been reported in patients with type $1 / 2$ diabetes [22]. The assessment of proximal tubule dysfunction by urinary $\alpha 1$-microglobulin allows the early diagnosis of diabetic nephropathy prior to the appearance of microalbuminuria and also predicts the progression of diabetic nephropathy. There is little literature about $\alpha 1$ microglobulin in nephrolithiasis patients, however [23]. Plasma angiotensinogen is filtered through glomerular capillaries, and urinary angiotensinogen is mainly derived from plasma. Subsequently, urinary angiotensinogen is largely removed via endocytotic uptake in tubules in a megalindependent manner. Endocytotic angiotensinogen is subsequently degraded and the contents of angiotensinogen in the proximal convoluted tubules highly correlated with plasma levels of angiotensinogen [24]. Thus, previous studies also support the idea that urinary excretion of angiotensinogen reflects not only abnormalities of glomerular filtration barrier but also proximal tubular functions. It has been reported that urinary angiotensinogen levels increase before 
glomerular injuries in the patients as well as in rodents [25]. Another limitation of our study is that no detailed data about hemodynamic index such as fractional excretion of sodium (FENa), perfusion flow rate (PFR), and even podocyte function parameters should be taken into account in future studies. As the literature reported, angiotensinogen was expressed in proximal tubular cells, and UA excretion was correlated with renal Ang II but not plasma Ang II; it was suggested that UA reflects renal Ang II production. Ang II will stimulate renal angiotensinogen synthesis, resulting in both elevated renal angiotensinogen levels and increased urinary angiotensinogen excretion, thus potentially creating a positive feed-forward loop. According to this concept, the rise in renal Ang II content following Ang II infusion involves de novo Ang II formation in the kidney from locally generated angiotensinogen [26]. The precise mechanism for the increment of renal de novo angiotensinogen generation in nephrolithiasis patients should be investigated in further studies.

In conclusion, we conclude in our study measurement of UA was a novel and useful marker for patients with nephrolithiasis. The investigation of UA suggests urologists to concern about previous neglected tubular injury in clinical kidney stone patients and greatly enhances our understanding of tubular injury in etiology of nephrolithiasis. Further validation of serum creatinine and urinary $\alpha 1$-microglobulin shows us the disease progression in nephrolithiasis cohort, which might require multicenter randomized study.

\section{Abbreviations}

UA: Urinary angiotensinogen

ACE: Angiotensin-converting enzyme

RAS: Renin-angiotensin system

Ang: Angiotensinogen

eGFR: Estimated glomerular filtration

Cr: Creatinine

NGAL: Neutrophil gelatinase-associated lipocalin

AKI: Acute kidney injury

CKD: Chronic kidney disease

KIM: Kidney injury molecule

ICU: Intensive care unit

ELISA: Enzyme-linked immunosorbent assay

FENa: Fractional excretion of sodium

PFR: Perfusion flow rate.

\section{Conflict of Interests}

The authors declared that there is no potential conflict of interests with respect to the authorship and/or publication of this paper.

\section{Authors' Contribution}

Wei Sun and Yuan Feng have equal first contributions in this work. Wei Sun, Min Liu, and Jun-Hua Zheng conceived and designed the experiments. Wei Sun, Jun-Hua Zheng, and Yuan Feng performed the experiments. Xu-Dong Yao and
Yun-Fei Xu analyzed the data. Wei Sun, Min Liu, Xu-Dong Yao, Bo Peng, Yun-Fei Xu, and Jun-Hua Zheng contributed with reagents, materials, and analysis tools. Wei Sun, Min Liu, and Jun-Hua Zheng wrote the paper.

\section{Acknowledgment}

This work was partially supported by Grants from the National Natural Science Foundation of China (no. 81000311 and no. 81270831).

\section{References}

[1] M. T. Keddis and A. D. Rule, "Nephrolithiasis and loss of kidney function," Current Opinion in Nephrology \& Hypertension, vol. 22, no. 4, pp. 390-396, 2013.

[2] A. Neisius and G. M. Preminger, "Stones in 2012: epidemiology, prevention and redefining therapeutic standards," Nature Reviews Urology, vol. 10, no. 2, pp. 75-77.

[3] S. R. Khan, "Role of renal epithelial cells in the initiation of calcium oxalate stones," Nephron-Experimental Nephrology, vol. 98, no. 2, pp. e55-e60, 2004.

[4] M. Moonen, V. Fraipont, L. Radermacher, C. Masset, É. Firre, and X. Warling, "Acute kidney injury: from concept to practice," Nephrologie et Therapeutique, vol. 7, no. 3, pp. 172-177, 2011.

[5] W. W. Batenburg and A. H. Danser, "(Pro)renin and its receptors: pathophysiological implications," Clinical Science, vol. 123, no. 3, pp. 121-133, 2012.

[6] L. G. Navar, M. C. Prieto, R. Satou, and H. Kobori, "Intrarenal angiotensin II and its contribution to the genesis of chronic hypertension," Current Opinion in Pharmacology, vol. 11, no. 2, pp. 180-186, 2011.

[7] H. Kobori, M. Nangaku, L. G. Navar, and A. Nishiyama, "The intrarenal renin-angiotensin system: from physiology to the pathobiology of hypertension and kidney disease," Pharmacological Reviews, vol. 59, no. 3, pp. 251-287, 2007.

[8] S. M. Kim, H. R. Jang, Y.-J. Lee et al., "Urinary angiotensinogen levels reflect the severity of renal histopathology in patients with chronic kidney disease," Clinical Nephrology, vol. 76, no. 2, pp. $117-123,2011$.

[9] W. H. Yiu, C.-J. Pan, R. A. Ruef et al., "Angiotensin mediates renal fibrosis in the nephropathy of glycogen storage disease type Ia," Kidney International, vol. 73, no. 6, pp. 716-723, 2008.

[10] M. Kamiyama, M. Urushihara, T. Morikawa et al., "Oxidative stress/angiotensinogen/renin-angiotensin system axis in patients with diabetic nephropathy," International Journal of Molecular Sciences, vol. 14, no. 11, pp. 23045-23062, 2013.

[11] J. L. Alge, N. Karakala, B. A. Neely, M. G. Janech, J. C. Velez, and J. M. Arthur, "Urinary angiotensinogen predicts adverse outcomes among acute kidney injury patients in the intensive care unit," Critical Care, vol. 17, no. 2, article R69, 2013.

[12] M. Decoster, J. C. Bigot, J. L. Carre et al., "Epidemiologic study of urinary calculi in Western France," Presse Medicale, vol. 31, no. 3, pp. 113-118, 2002.

[13] T. Terami, J. Wada, K. Inoue et al., "Urinary angiotensinogen is a marker for tubular injuries in patients with type 2 diabetes," International Journal of Nephrology and Renovascular Disease, vol. 6, pp. 233-240, 2013. 
[14] H. Kobori, L. M. Harrison-Bernard, and L. G. Navar, "Urinary excretion of angiotensinogen reflects intrarenal angiotensinogen production," Kidney International, vol. 61, no. 2, pp. 579$585,2002$.

[15] H. Kobori, A. Nishiyama, L. M. Harrison-Bernard, and L. Gabriel Navar, "Urinary angiotensinogen as an indicator of intrarenal angiotensin status in hypertension," Hypertension, vol. 41, no. 1, pp. 42-49, 2003.

[16] H. M. Siragy and R. M. Carey, "Role of the intrarenal reninangiotensin-aldosterone system in chronic kidney disease," American Journal of Nephrology, vol. 31, no. 6, pp. 541-550, 2010.

[17] T. W. Hsu, J. S. Liu, S. C. Hung et al., "Renoprotective effect of Renin-Angiotensin-Aldosterone system blockade in patients with predialysis advanced chronic kidney disease, hypertension, and anemia," Journal of the American Medical Association Internal Medicine, vol. 174, no. 3, pp. 347-354, 2014.

[18] J. L. Alge, N. Karakala, B. A. Neely et al., "Urinary angiotensinogen and risk of severe AKI," Clinical Journal of the American Society of Nephrology, vol. 8, no. 2, pp. 184-193, 2013.

[19] L. C. Roksnoer, K. Verdonk, A. H. van den Meiracker, E. J. Hoorn, R. Zietse, and A. H. Danser, "Urinary markers of intrarenal renin-angiotensin system activity in vivo," Current Hypertension Reports, vol. 15, no. 2, pp. 81-88, 2013.

[20] M. van den Heuvel, W. W. Batenburg, S. Jainandunsing et al., "Urinary renin, but not angiotensinogen or aldosterone, reflects the renal renin-angiotensin-aldosterone system activity and the efficacy of renin-angiotensin-aldosterone system blockade in the kidney," Journal of Hypertension, vol. 29, no. 11, pp. 21472155, 2011.

[21] C.-Y. Hong, K. Hughes, K.-S. Chia, V. Ng, and S.-L. Ling, "Urinary $\alpha 1$-microglobulin as a marker of nephropathy in type 2 diabetic Asian subjects in Singapore," Diabetes Care, vol. 26, no. 2, pp. 338-342, 2003.

[22] L. Petrica, M. Petrica, A. Vlad et al., "Proximal tubule dysfunction is dissociated from endothelial dysfunction in normoalbuminuric patients with type 2 diabetes mellitus: a cross-sectional study," Nephron-Clinical Practice, vol. 118, no. 2, pp. c155-c164, 2011.

[23] M. Igci, A. Arslan, Y. Z. Igci et al., "Bikunin and $\alpha 1-$ microglobulin/bikunin precursor (AMBP) gene mutational screening in patients with kidney stones: a case-control study," Scandinavian Journal of Urology and Nephrology, vol. 44, no. 6, pp. 413-419, 2010.

[24] J. P. Richoux, J. L. Cordonnier, J. Bouhnik et al., "Immunocytochemical localization of angiotensinogen in rat liver and kidney," Cell and Tissue Research, vol. 233, no. 2, pp. 439-451, 1983.

[25] M. Kamiyama, A. Zsombok, and H. Kobori, "Urinary angiotensinogen as a novel early biomarker of intrarenal renin-angiotensin system activation in experimental type 1 diabetes," Journal of Pharmacological Sciences, vol. 119, no. 4, pp. 314-323, 2012.

[26] R. A. Gonzalez-Villalobos, T. Janjoulia, N. K. Fletcher et al., "The absence of intrarenal ACE protects against hypertension," The Journal of Clinical Investigation, vol. 123, no. 5, pp. 2011-2023, 2011. 


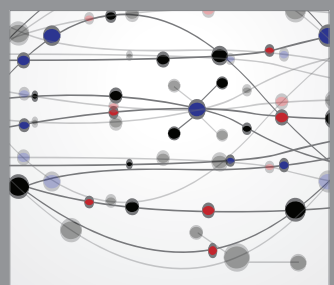

The Scientific World Journal
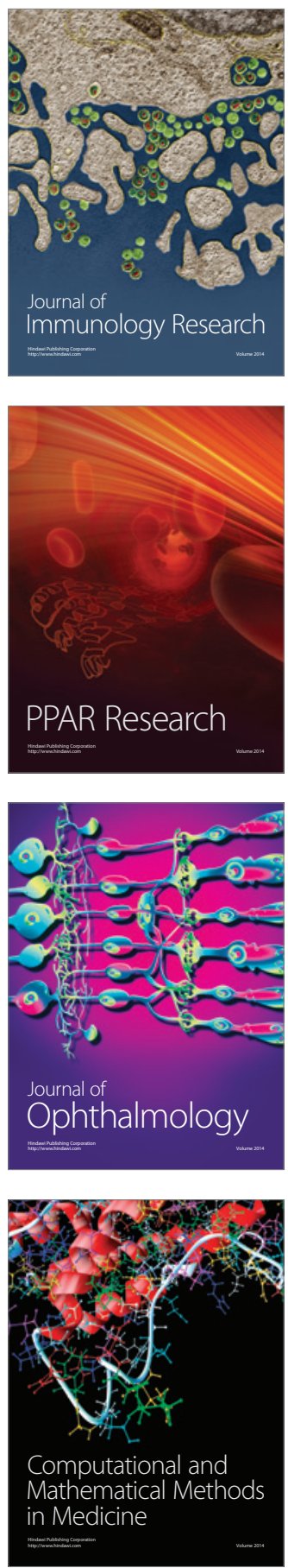

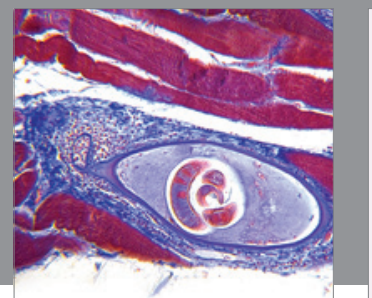

Gastroenterology

Research and Practice
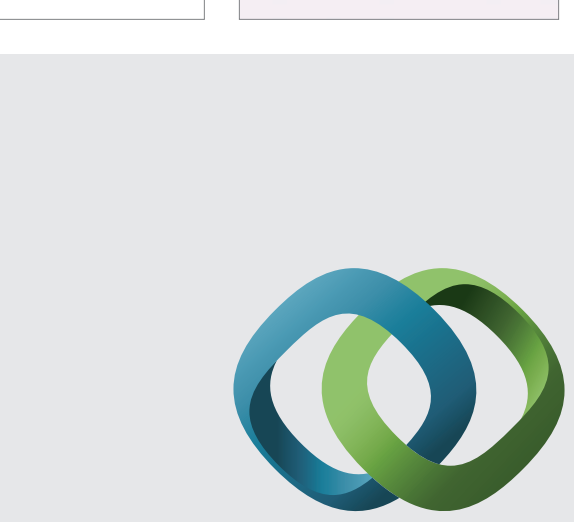

\section{Hindawi}

Submit your manuscripts at

http://www.hindawi.com
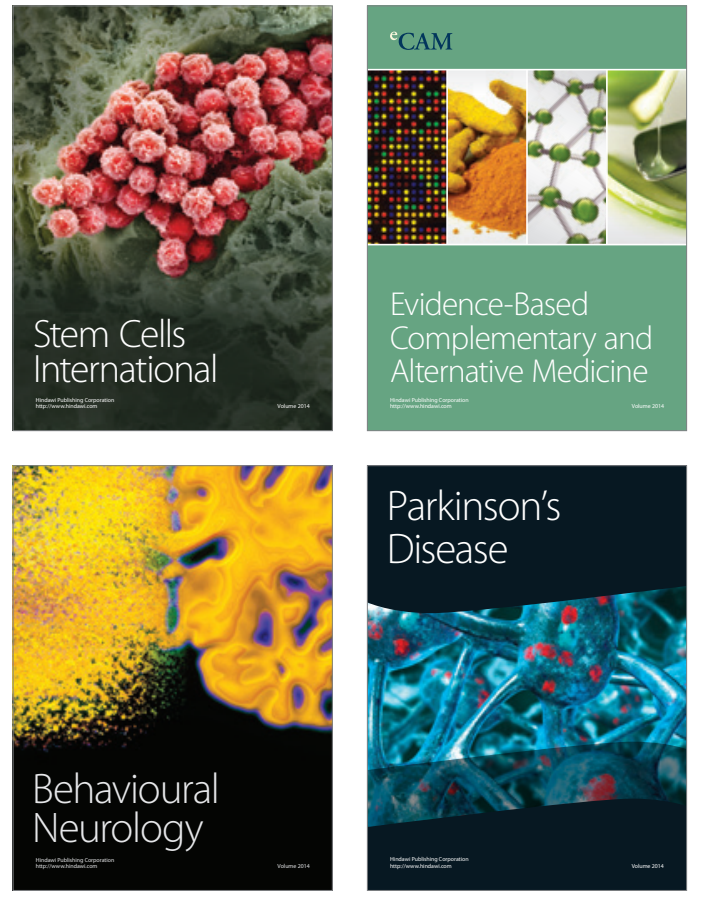
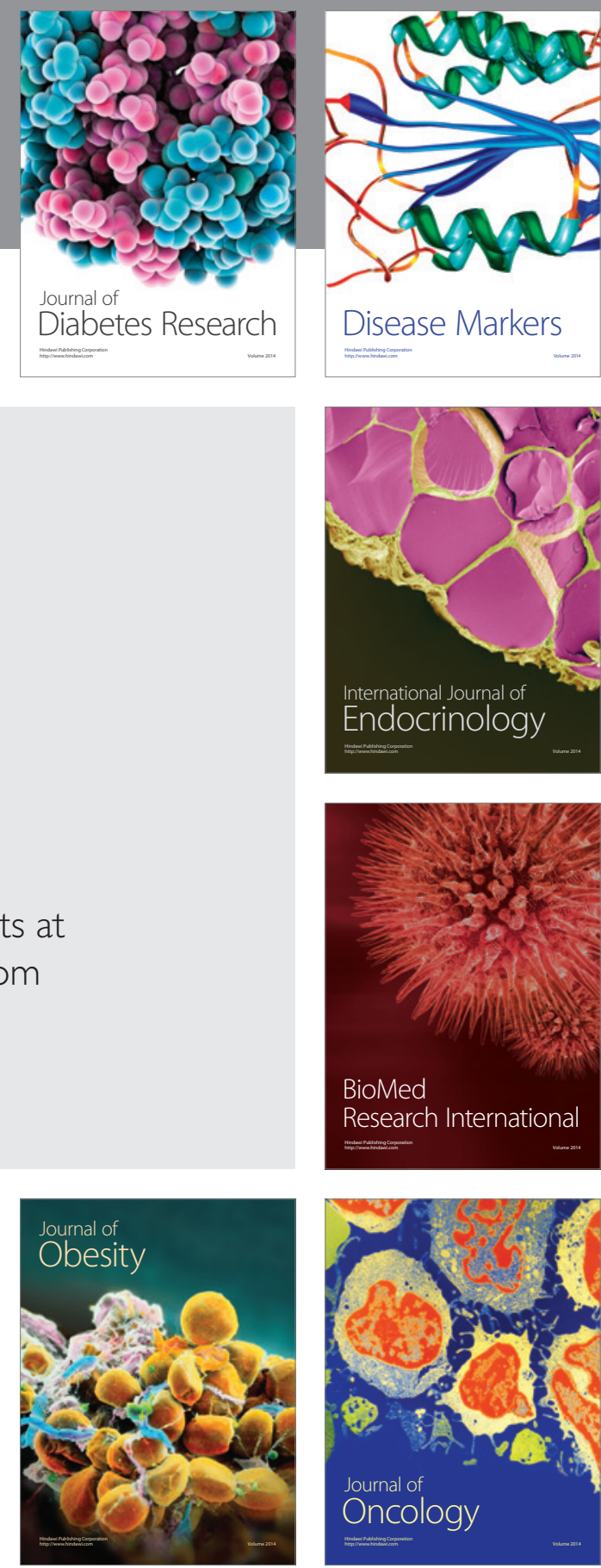

Disease Markers
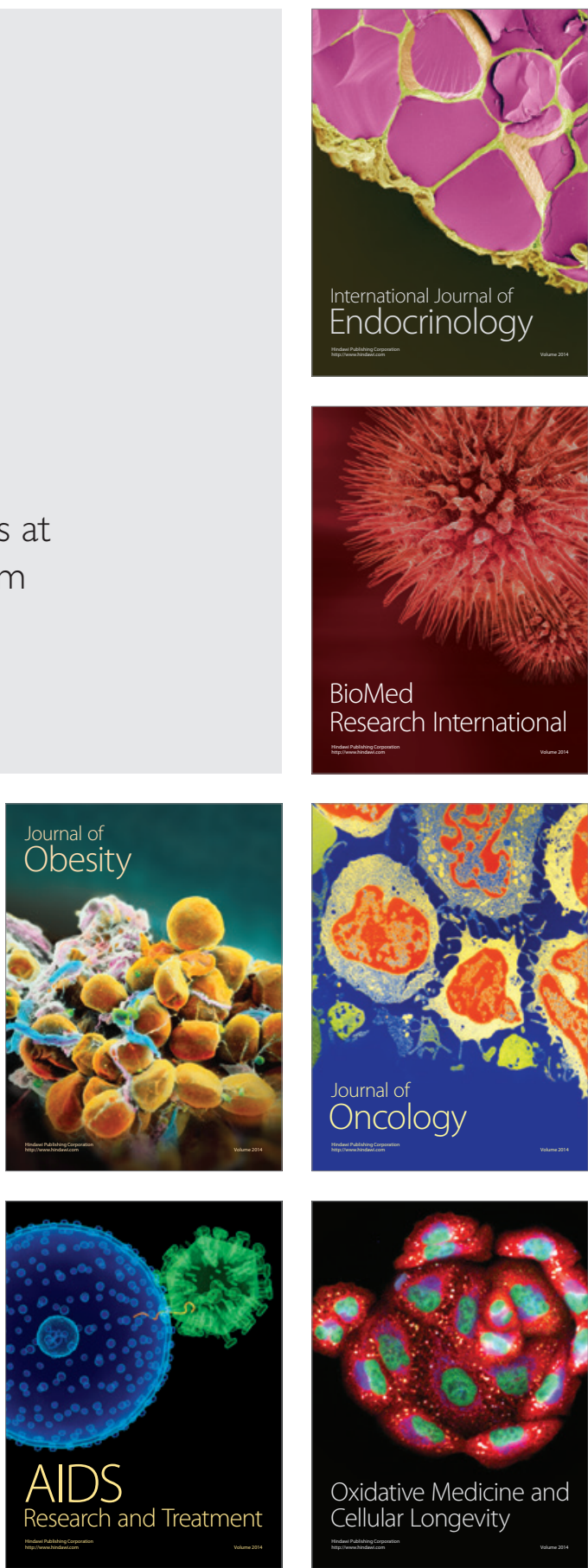